\title{
CRÉATION CONTRE SCIENCE EN DESIGN, LES CONDITIONS D'UN VRAI DÉBAT : RÉPONSE À LYSIANNE LÉCHOT HIRT
}

Philippe Gauthier

\author{
Presses Universitaires de France | « Sciences du Design »
}

$2015 / 2 n^{\circ} 2 \mid$ pages 65 à 70

ISSN 2428-3711

Article disponible en ligne à l'adresse :

http://www.cairn.info/revue-sciences-du-design-2015-2-page-65.htm

\section{!Pour citer cet article :}

Philippe Gauthier, «Création contre science en design, les conditions d'un vrai débat : réponse à Lysianne Léchot Hirt », Sciences du Design 2015/2 (n²), p. 65-70.

Distribution électronique Cairn.info pour Presses Universitaires de France.

(C) Presses Universitaires de France. Tous droits réservés pour tous pays.

La reproduction ou représentation de cet article, notamment par photocopie, n'est autorisée que dans les limites des conditions générales d'utilisation du site ou, le cas échéant, des conditions générales de la licence souscrite par votre établissement. Toute autre reproduction ou représentation, en tout ou partie, sous quelque forme et de quelque manière que ce soit, est interdite sauf accord préalable et écrit de l'éditeur, en dehors des cas prévus par la législation en vigueur en France. Il est précisé que son stockage dans une base de données est également interdit. 


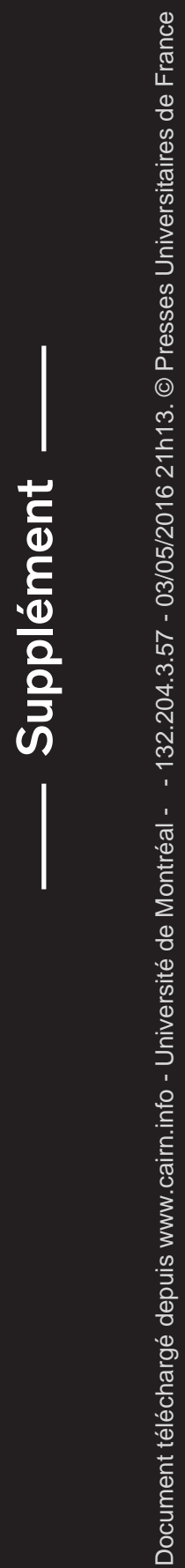


Professeur à l'École de design de la Faculté d'aménagement, Directeur du groupe de recherche Design $n$ société, Université de Montréal

philippe.gauthier.2@umontreal.ca

\section{Création contre} science en design, les conditions d'un vrai débat: réponse à Lysianne Léchot Hirt 


\section{Introduction}

Le premier numéro de la revue Sciences du Design accueillait un article de Lysianne Léchot-Hirt qui, je crois, mérite quelques mises au point. L'article, intitulé "Recherche-création en design à plein régime: un constat, un manifeste, un programme " (Léchot-Hirt, 2015), se présente comme un réquisitoire contre une certaine forme de recherche que l'auteure de l'article qualifie de "scientiste». Je me porterai donc à la défense de cette forme de recherche. Non pas que ses protagonistes aient besoin qu'on les défende (ils n'en demandent peut-être pas tant), mais il me semble important 1) de montrer les dangers de certains des arguments utilisés par l'auteure et 2) de proposer un programme alternatif à celui évoqué.

\section{1. - Les termes de la confrontation}

On aurait pu espérer que dans le cadre d'un article publié dans une revue savante, les concepts centraux utilisés par son auteure aient fait l'objet d'un travail de définition permettant de limiter toute équivoque à leur propos. De même, s'agissant de confronter deux réalités de la pratique de la recherche en design, on aurait pu s'attendre à ce que Léchot-Hirt rende mieux compte de l'ensemble des arguments, idées et propositions que cette confrontation a pu faire naître au cours des dernières années. Et ils sont nombreux. Enfin, les cas évoqués par l'auteure pour appuyer ses affirmations et la stratégie argumentative employée exposent le propos à un dangereux risque de complaisance.

Dans le cadre de son article, l'auteure oppose ainsi ce qu'elle conçoit comme deux modalités de la recherche en design: la recherche-création d'un côté et la recherche scientiste à laquelle elle associe la recherche-projet, de l'autre. Cette confrontation, du moins les arguments avancés par l'auteure, renvoie à un débat ancien mais toujours d'actualité dans notre discipline, mais ailleurs également, notamment en sciences sociales et humaines. Afin de rester dans les limites d'un simple commentaire, je proposerais de résumer ce débat par la question de la place du sujet dans la production de connaissances, du rapport entre subjectivité et véridicité. Dans le champ du design, ce débat se double de la concurrence entre deux traditions de pratique qui peinent toujours à se réconcilier : la tradition des arts décoratifs qui s'est développée sous l'autorité des beaux-arts et la tradition du design industriel dans laquelle le marketing a joué un rôle majeur. Ces deux traditions ont dessiné des éthiques du designer fort différentes auxquelles la confrontation développée par Léchot-Hirt me semble faire écho. Or, ici, bien que l'auteure affirme vouloir "creuser la querelle idéologique afin de dégager une compréhension du concept permettant un passage à l'action ", je ne crois pas que son propos parvienne à apporter un éclairage nouveau à la situation évoquée et, à défaut d'offrir une analyse compréhensive des enjeux de cette vieille querelle idéologique, il me semble difficile de parvenir un jour à l'apaiser. 


\section{2. - Le cryptoconstructivisme à la rescousse de la création}

Le concept de recherche-projet, qui fait l'objet du réquisitoire de l'auteure, est rapidement substitué par celui de recherche scientiste. Cette substitution de la recherche-projet à une forme de "isme ", réduit à un simple simulacre le travail des chercheurs s'engageant dans la voie de la recherche-projet. Or, il ne s'agit pas de n'importe quel simulacre. En qualifiant de scientiste l'engagement dans cette voie, et en convoquant la vulgate latourienne pour dénoncer ce qui n'est au fond que de simples travers bien connus de la communauté de la recherche, toutes disciplines confondues, c'est l'exigence d'universalité des connaissances à laquelle tentent de se plier certains chercheurs en design qui est ici remise en cause. Ainsi, tout effort pour engager le design dans un projet scientifique ne relèverait, au mieux, que d'une posture et, à ce jeu, la recherche-projet, scientiste, n'aurait aucun mérite particulier par rapport à la recherche-création («En effet! » diraient les mauvaises langues). On ne saurait contredire l'auteure sur les dangers que représente l'adoption par les membres de la communauté de la recherche en design de la pose scientiste. L'exigence de véridicité, d'universalité et de validité ne peut souffrir aucun des faux-semblants que, malheureusement, la bureaucratisation de la recherche universitaire tend à considérer comme l'essence même de la quête de connaissances.

Toutefois, il ne faut pas se méprendre. La portée de l'argument de l'auteure déborde largement ce seul plan qu'on pourrait qualifier d'administratif et sur lequel une grande partie de la communauté de la recherche pourra s'entendre. Forte de l'argument constructiviste, c'est sur le plan épistémologique qu'elle souhaite démonter la baudruche de la recherche-projet.

Selon la vulgate constructiviste, la science moderne aurait réduit la quête de vérités universelles à l'adoption de conventions entre chercheurs, faisant en sorte que la seule preuve dont aurait besoin le designer pour asseoir la validité des connaissances qu'il propose serait l'intérêt qu'y trouveraient ses pairs. Incidemment, Léchot-Hirt développe une preuve empirique de la surévaluation de la recherche-projet, basée sur sa propre expérience de la recherche. L'adoption des standards décatis de la présentation scientifique à laquelle l'auteure réduit les conditions de scientificité d'une proposition observées par les protagonistes de la recherche scientiste, serait le déterminant fondamental de ce profond ennui qui caractérise son expérience-usager lors des nombreux symposiums auxquels elle assiste. À l'inverse, la recherche-création, elle, la captive. Cet effort ethnographique louable ne peut cacher la fragilité de la technique utilisée par l'auteure pour tirer un échantillon probant parmi les nombreux exemples de recherche-projets et de recherche-créations existants. Cet échantillonnage aurait dû tenir compte, entre autres, du fait que la recherche scientiste souffre d'une très grande pression administrative à la production. Le phénomène du publish or perish pousse une grande partie de la communauté à privilégier la quantité de recherches produites au détriment, bien évidemment, de la qualité ${ }^{01}$. Dans ce cadre, les symposiums se présentent comme des moments clés, parfaitement instrumentalisables pour des visées de carrière universitaire, lors desquels des centaines de papers sont alignés dans des sessions de présentation parallèles. Ce sont les travers de la bureaucratisation de la recherche que démontre l'ennui inspiré par les symposiums, pas l'ineffectivité des conventions scientifiques qui, comme en convient elle-même Léchot-Hirt, semblent résister à toutes les tentatives (un-conference, $x$-camp, $x$-jam, $x$-athon) pour les transformer. Dans ce cadre favorable à la multiplication des mauvaises recherches scientistes, l'étude de cas aurait été plus pertinente que la construction de séries d'événements dont la
01.

II s'agit plus précisément de multiplier la quantité des indices (accounts) de recherches dont le nombre a un effet direct sur la carrière professorale, mais qui est parfois totalement indépendant de la qualité de la recherche produite. 
02.

En dépit des réserves émises par l'auteure envers les rapprochements effectuées depuis quelques années entre la recherche en design et les sciences sociales et humaines, je pousserais ici l'affront en convoquant un ténor de la sociologie pour tenter de comprendre ce qui se trame derrière un tel déni de légitimité lancé à la figure de la science et du monde universitaire. En effet, Pierre Bourdieu y aurait sans doute vu une simple stratégie développée par une certaine classe de designers "dominés", ou se sentant dominés, souhaitant s'accaparer à peu de frais le capital symbolique que procure le titre de professeur-chercheur d'université dans le champ social. disproportion ne peut mener qu'à un biais de sélection. Au demeurant, il aurait été essentiel de vérifier si le fait que la recherche scientiste paraisse si majoritairement insignifiante ne s'explique pas simplement par le fait qu'il y a plus de recherches scientistes que de recherche-créations. Dans ce cadre, la sélection complaisante de quelques exemples faisant autorité parmi les designers engagés dans des démarches dites de recherche-création tient de la supercherie.

Le problème est assez grave car sur le plan épistémologique, le cryptoconstructivisme de l'auteure parvient non seulement à discréditer la rechercheprojet pour cause d'affectation scientiste par ses protagonistes, mais aussi l'ensemble du projet scientifique engagé par des générations entières de chercheurs, et pas seulement de chercheurs en design ${ }^{\mathbf{0 2}}$.

\section{3. - Rendre justice à des pratiques distinctes}

Au-delà de ce réquisitoire contre l'organisation de la science à laquelle est confrontée une partie de la communauté de la recherche en design, l'auteure vise à affirmer la légitimité de la recherche-création sur le plan épistémologique. Réduisant ce plan épistémologique à sa plus simple expression faite d'événements institués, de conventions circonstancielles et de formes canoniques d'exposition, l'auteure a beau jeu de poser la question «En quoi la recherche-création pose-t-elle problème? " Poser cette question dans ce cadre c'est sous-entendre que la réflexion épistémologique sur la validité de l'une ou l'autre forme de recherche est elle-même vaine. C'est supposer que la question ne se pose pas. Or, il me semble qu'un des devoirs d'une communauté prétendant proposer des connaissances qui puissent faire avancer la compréhension du monde dans lequel on vit, est de faire la preuve de ce que ses membres avancent en veillant à toujours permettre un examen scrupuleux des modalités de construction des assertions sur le monde qu'ils produisent. Ainsi, contrairement à ce que l'auteure suggère, aucun chercheur honnête ne peut jamais échapper à la question «en quoi la recherche-[création/projet/scientiste] pose problème? ». Cette question est la concrétisation même de l'hypothèse sceptique au cœur de tout effort épistémologique (Tiercelin, 2005). Examiner les fondements de ses assertions, en rendre compte, en débattre et donc se poser la question " en quoi mon travail pose-t-il problème? ? est une des tâches fondamentales, toujours à refaire, exigées par la recherche de connaissances. Tenter d'échapper à cette question pour soi sous prétexte que l'effort d'autrui reste toujours insatisfaisant, c'est faillir à ses propres engagements de chercheurs.

Par conséquent, j'aimerais proposer que s'il y a une frontière à tracer entre deux camps, elle est donc bien entre la pratique de la recherche et la pratique du design (ou de la création). Les deux activités sont parfaitement légitimes dans leur monde respectif et, en tant que constituantes essentielles de la communauté de pratique du design, elles doivent pouvoir dialoguer et s'enrichir. Mais les deux activités ne sont pas de même nature. Faire de la création ou du design de très grande qualité est extrêmement exigeant. Toutefois, ces exigences ne concernent pas le travail épistémologique qui impose ses propres contraintes à la recherche, contraintes qu'on n'apprend pas à satisfaire en pratiquant le design. Apposer l'étiquette "recherche " à une activité de création, de design ou de médiation sociale ne suffit pas à en changer la nature. Seul l'effort d'élucidation épistémologique peut y parvenir. Cette irréductibilité de la pratique du design, d'un 
côté, et de la pratique de la recherche de l'autre, explique d'ailleurs pourquoi un chercheur en design peut être un bien mauvais designer : il ne s'agit pas du même travail!

\section{4. - Un programme: la revue Sciences du Design}

La vivacité de la réflexion épistémologique prenant forme dans une communauté de pratique doit être considérée comme la condition sine qua non de la recherche dans cette communauté. Le but d'une revue portant le titre Sciences du Design devrait justement être de susciter cette réflexion auprès de l'ensemble des membres de notre communauté. De ce point de vue, la question «En quoi la recherche-création pose-t-elle problème ? " est une question éminemment pertinente qui devrait occuper le plus clair de la réflexion épistémologique des chercheurs qui croient en la recherche-création. Ce devoir réflexif dessine un vrai programme dans lequel ces chercheurs devront tôt ou tard s'engager. La revue Sciences du Design, qui, comme de nombreuses autres revues savantes, tente d'établir une relation critique raisonnée avec les conventions de la recherche, est certainement un des lieux privilégiés où les protagonistes de cette forme de recherche peuvent montrer leur attachement à la connaissance en intégrant le débat déjà houleux et que l'on souhaite fécond de la réflexion épistémologique en design.

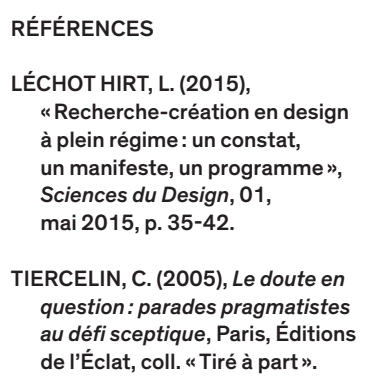

